\title{
FIBONACCI-EVEN NUMBERS: BINARY ADDITIVE PROBLEM, DISTRIBUTION OVER PROGRESSIONS, AND SPECTRUM
}

\author{
V. G. ZHURAVLEV
}

Abstract. The representations $\vec{N}_{1}+\vec{N}_{2}=D$ of a natural number $D$ as the sum of two Fibonacci-even numbers $\vec{N}_{i}=F_{1} \circ N_{i}$, where $\circ$ is the circular Fibonacci multiplication, are considered. For the number $s(D)$ of solutions, the asymptotic formula $s(D)=c(D) D+r(D)$ is proved; here $c(D)$ is a continuous, piecewise linear function and the remainder $r(D)$ satisfies the inequality

$$
|r(D)| \leq 5+\left(\frac{1}{\ln (1 / \tau)}+\frac{1}{\ln 2}\right) \ln D
$$

where $\tau$ is the golden section.

The problem concerning the distribution of Fibonacci-even numbers $\vec{N}$ over arithmetic progressions $\vec{N} \equiv r \bmod d$ is also studied. Let $l_{F_{1}}(d, r, X)$ be the number of $N ' s, 0 \leq N \leq X$, satisfying the above congruence. Then the asymptotic formula

$$
l_{F_{1}}(d, r, X)=\frac{X}{d}+c(d) \ln X
$$

is true, where $c(d)=O(d \ln d)$ and the constant in $O$ does not depend on $X, d$, or $r$. In particular, this formula implies the uniformity of the distribution of the Fibonacci-even numbers over progressions for all differences $d=O\left(\frac{X^{1 / 2}}{\ln X}\right)$.

The set $\overrightarrow{\mathbb{Z}}$ of Fibonacci-even numbers is an integral modification of the well-known one-dimensional Fibonacci quasilattice $\mathcal{F}$. Like $\mathcal{F}$, the set $\overrightarrow{\mathbb{Z}}$ is a quasilattice, but it is not a model set. However, it is shown that the spectra $\Lambda_{\mathcal{F}}$ and $\Lambda_{\overrightarrow{\mathbb{Z}}}$ coincide up to a scale factor $\nu=1+\tau^{2}$, and an explicit formula is obtained for the structural amplitudes $f_{\vec{Z}}(\lambda)$, where $\lambda=a+b \tau$ lies in the spectrum:

$$
f_{\overrightarrow{\mathbb{Z}}}(\lambda)=\frac{\sin (\pi b \tau)}{\pi b \tau} \exp (-3 \pi i b \tau)
$$

\section{INTRODUCTION}

0.1. The one-dimensional Fibonacci quasilattice $\mathcal{F}$. We define a map $\delta$ of the set of rational integers $\mathbb{Z}$ to the half-open interval $J=[-1, \tau)$ by setting

$$
\mathbb{Z} \stackrel{\delta}{\longrightarrow} J: N \mapsto \delta(N)=N-[(N+1) \tau] \widetilde{\tau},
$$

where $\tau=\frac{-1+\sqrt{5}}{2}$ is the golden section, $\widetilde{\tau}=\tau+1$ and $[x]$ is the integral part of $x$. With the help of the map $\delta$, we define the subset

$$
\mathcal{F}=\delta^{\prime}(\mathbb{Z})=\left\{\delta^{\prime}(N)=N+[(N+1) \tau] \tau ; N \in \mathbb{Z}\right\}
$$

2000 Mathematics Subject Classification. Primary 06A11.

Key words and phrases. Fibonacci-even numbers, Fibonacci quasilattices, Fibonacci circular multiplication, Diophantine equations, spectrum.

Supported by RFBR (grant no. 05-01-00435). 
of the set of real numbers $\mathbb{R}$. Here the prime ' means conjugation in the real quadratic field $\mathbb{Q}(\sqrt{5})$. The set $\mathcal{F}$ is a one-dimensional Fibonacci quasilattice (see Figure 1) and is a model set (see, e.g., [17]-[19]), because the half-open interval $J$ is a window for it. Thus, the quasilattice $\mathcal{F}$ admits another equivalent definition:

$$
\mathcal{F}=\left\{x \in \mathbb{Z}[\tau] ; x^{\prime} \in J\right\} .
$$

Here $\mathbb{Z}[\tau]=\{a+b \tau ; a, b \in \mathbb{Z}\}$ is the Fibonacci ring, or the ring of integers in the field $\mathbb{Q}(\sqrt{5})$.

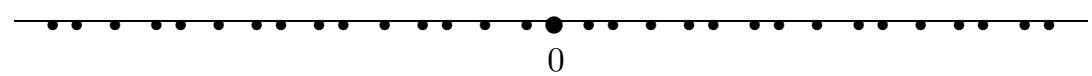

Figure 1. One-dimensional Fibonacci quasilattice $\mathcal{F}$.

The above quasilattice possesses a number of interesting properties (see [3, 5]). For example, the quasilattice $\mathcal{F}$ has a rich semigroup of self-similarities $\mathcal{S}=\delta^{\prime}\left(\mathbb{Z}_{\tau}\right)$, where $\mathbb{Z}_{\tau}$ is the set of all $N \in \mathbb{Z}$ with $\left|\delta^{\prime}(N)\right|<\tau$. In its turn, the semigroup $\mathcal{S}$ itself is a quasilattice in $\mathcal{F}$. For any $\lambda=\delta^{\prime}(N)$ in $\mathcal{S}$, we have the inclusion

$$
\lambda \cdot \mathcal{F} \subset \mathcal{F} .
$$

If we take $N=1$, then we obtain similarity with the coefficient $\lambda=\delta^{\prime}(1)=\widetilde{\tau}$, which is the principal unit of the Fibonacci ring $\mathbb{Z}[\tau]$. The self-similarity (0.4) is an inflation of the quasilattice $\mathcal{F}$ (see [4]).

0.2. Circular Fibonacci multiplication. An attempt to explain the inclusion

$$
\delta^{\prime}\left(\mathbb{Z}_{\tau}\right) \cdot \delta^{\prime}(\mathbb{Z}) \subset \delta^{\prime}(\mathbb{Z})
$$

leads to the notion of the circular Fibonacci multiplication $N_{1} \circ N_{2}$. Let

$$
F_{1}=1, F_{2}=2, \ldots, F_{n}=F_{n-1}+F_{n-2}, \ldots
$$

be the Fibonacci numbers. Then any natural number $N$ expands uniquely in a finite sum of the form

$$
N=\varepsilon_{1} F_{1}+\varepsilon_{2} F_{2}+\cdots+\varepsilon_{n} F_{n}+\cdots=\sum_{n \geq 1} \varepsilon_{n}(N) F_{n},
$$

where the coefficients $\varepsilon_{n}$ take the values 0 or 1 and possess the cancellation property $\varepsilon_{n} \varepsilon_{n+1}=0$ for all $n \geq 1$. The number of nonzero terms $k(N)$ in the expansion (0.6) is called the length of the number $N$ in the Fibonacci number system. In this number system, the o-product $N_{1} \circ N_{2}$ is calculated by the formula

$$
N_{1} \circ N_{2}=\sum_{n \geq 1} \sum_{m \geq 1} \varepsilon_{n}\left(N_{1}\right) \varepsilon_{m}\left(N_{2}\right) F_{n+m},
$$

where $N_{1}, N_{2} \in \mathbb{N}$. The expansion (0.7) may fail to be a canonical expansion of the form (0.6). In $[3$, it was proved that o-multiplication can be written via the function $[x]$ in the form

$$
N_{1} \circ N_{2}=N_{1} N_{2}+\left[\left(N_{1}+1\right) \tau\right]\left[\left(N_{2}+1\right) \tau\right],
$$

and moreover, it is linked with the $\delta$-map (0.1) by the relation

$$
\delta\left(N_{1} \circ N_{2}\right)=\delta\left(N_{1}\right) \cdot \delta\left(N_{2}\right)+\left\{\begin{array}{lll}
0 & \text { if } & \delta\left(N_{1}\right) \cdot \delta\left(N_{2}\right) \in J, \\
-\widetilde{\tau} & \text { if } & \delta\left(N_{1}\right) \cdot \delta\left(N_{2}\right) \notin J .
\end{array}\right.
$$

Formula (0.8) makes it possible to extend o-multiplication from the set of natural numbers to the entire set of rational integers $\mathbb{Z}$, and formula (0.9) remains valid under this 
extension. For the first time, o-multiplication was defined by Matiyasevich in [8, 9, and later it appeared independently in the paper [15] by D. Knuth.

0.3. Fibonacci-even numbers. With the help of o-multiplication, one can define another class of one-dimensional Fibonacci quasilattices:

$$
\mathcal{F}_{A}=A \circ \mathbb{Z}=\{A \circ N ; N \in \mathbb{Z}\},
$$

where $A$ is any nonzero rational integer and o-multiplication is defined by formula (0.8). It is natural to call such quasilattices $\mathcal{F}_{A}$ o-progressions in the set $\mathbb{Z}$. Note that they are not model sets. The quasilattices $\mathcal{F}$ and $\mathcal{F}_{A}$ are combinatorially indistinguishable (cf. Figures 1 and 2), and for this reason they have the same complexity functions (see [12]). Our purpose in the present paper is to study the arithmetic, geometric, combinatorial, and spectral properties of the quasilattices $\mathcal{F}_{A}$. Previously, similar properties of the quasilattice $\mathcal{F}$ were studied in [3, 5 .

As a model, we choose the simplest quasilattice $\mathcal{F}_{F_{1}}=F_{1} \circ \mathbb{Z}$. It can be obtained by the shift

$$
F_{1} \circ N=\sum_{n \geq 1} \varepsilon_{n}(N) F_{n+1}
$$

of numbers $N$ written in the Fibonacci number system (0.6). Therefore, it is convenient to introduce the notation

$$
\overrightarrow{\mathbb{Z}}=\left\{\vec{N}=F_{1} \circ N ; N \in \mathbb{Z}\right\} .
$$

Formula (0.11) implies that a natural number $N$ belongs to $\overrightarrow{\mathbb{Z}}$ if and only if $\varepsilon_{1}(N)=0$. For this reason, $\overrightarrow{\mathbb{Z}}$ is called the set of Fibonacci-even numbers. By using formula (0.8), the shifted numbers $\vec{N}$ can be written in the explicit form:

$$
\vec{N}=N+[(N+1) \tau]
$$

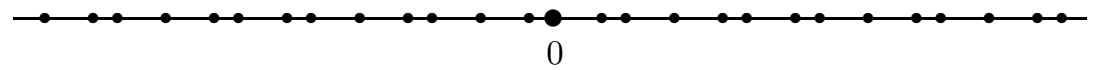

Figure 2. The quasilattice $\overrightarrow{\mathbb{Z}}$ of Fibonacci-even numbers.

0.4. Binary additive problem. The first problem to be considered concerns the representation of a natural number $D$ as a sum

$$
\vec{N}_{1}+\vec{N}_{2}=D
$$

where $N_{i}=0,1,2, \ldots$ Let $s(D)$ be the number of solutions of the Diophantine equation (0.14). In Theorem 1.1 we prove an asymptotic formula for $s(D)$ :

$$
s(D)=c(D) D+r(D),
$$

where $r(D)$ satisfies the inequality

$$
|r(D)| \leq 5+\left(\frac{1}{\ln \widetilde{\tau}}+\frac{1}{\ln 2}\right) \ln D,
$$

the coefficient is a continuous and piecewise linear function of $\delta(D)$, and its graph is presented in Figure 3. Analytically, the function $c(D)$ is given in formula (1.16).

In particular, the representability of any natural number $D$ as a sum of two nonnegative Fibonacci-even numbers follows from formula (0.15). 


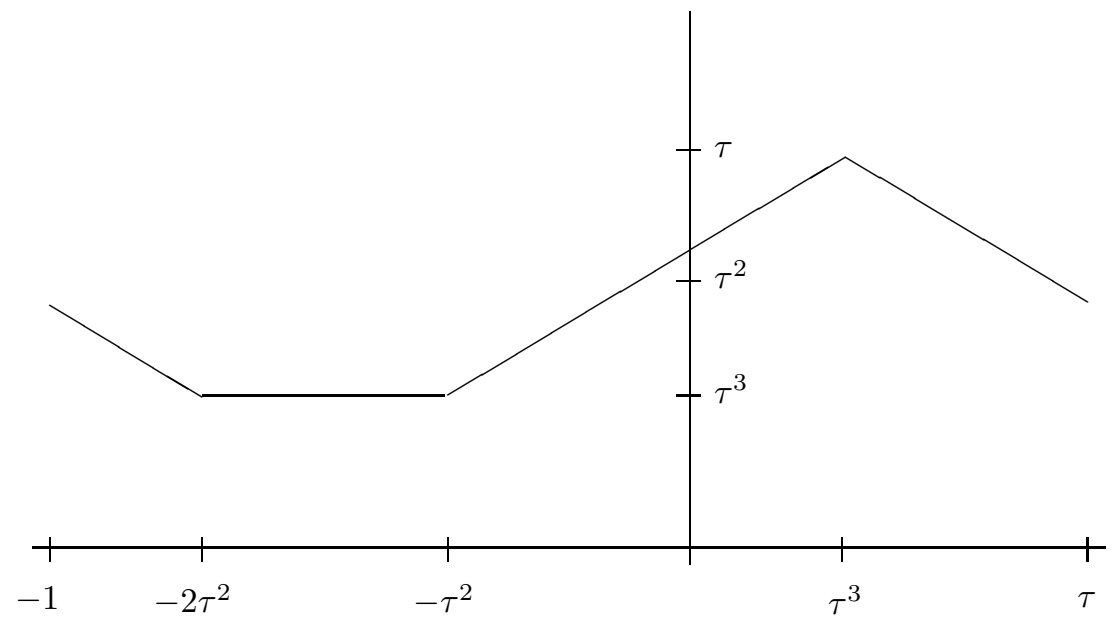

Figure 3. The graph of the function $c(D)$, where values of $\delta(D)$ are plotted on the axis $O X$.

0.5. Precise formulas for $s(D)$. The function $c(D)$ is constant on the half-open interval $\left[-2 \tau^{2},-\tau^{2}\right.$ ) and takes the value $\tau^{3}$ on this interval (see Figure 3). An explanation of this fact is given in Theorem 2.1, in which it is proved that the number $s(D)$ of solutions can be evaluated by the explicit formula

$$
s(D)=\left[D \tau^{3}\right]
$$

for all $D \geq 1$ such that $\delta(D)$ belongs to the half-open interval $\left[-2 \tau^{2},-\tau^{2}\right)$. Comparing formulas (0.15) and (0.16), we obtain the relation

$$
r(D)=-\left\{D \tau^{3}\right\},
$$

and thus, for $D$ as mentioned above, the remainder $r(D)$ is bounded:

$$
|r(D)|<1 .
$$

If we take the Fibonacci numbers $F_{n}, n \geq 3$, for the role of $D$, then in Theorem 2.2 we have the formula

$$
s(D)=F_{n-1}-F_{n-4}+2 e(n),
$$

where $e(n)$ is equal to 1 or 0 for $n$ even or odd, respectively. This formula also implies the boundedness of the remainder $r(D)$.

0.6. Distribution over progressions. The problem concerning the uniformity of the distribution of Fibonacci-even numbers over progressions is considered in $\S 3$. Let $l_{F_{1}}(d, r, X)$ denote the number of Fibonacci-even numbers $\vec{N}_{1}=F_{1} \circ N_{1}$ such that $0 \leq N_{1} \leq X$ and $\vec{N}_{1} \equiv r \bmod d$, where $d$ and $r$ are arbitrary rational integers and $d>1$. Then, by Theorem 3.1, the following asymptotic formula is valid for $l_{F_{1}}(d, r, X)$ :

$$
l_{F_{1}}(d, r, X)=\frac{X}{d}+c(d) \ln X
$$

as $X \rightarrow+\infty$, where $c(d)=O(d \ln d)$ and the constant in $O$ does not depend on $X, d$, or $r$. In this case, to prove formula (0.18), the equation

$$
\vec{N}_{1}-d N_{2}=r
$$

is used instead of the Diophantine equation (0.14). We note that (0.18) implies the uniformity of the distribution of Fibonacci-even numbers over progressions for all differences $d=O\left(\frac{X^{1 / 2}}{\ln X}\right)$. 
0.7. Spectrum. The spectrum of Fibonacci-even numbers $\overrightarrow{\mathbb{Z}}$ is the maximal subset $\Lambda_{\overrightarrow{\mathbb{Z}}}$ in $\mathbb{R}$ for which the condition $f_{\overrightarrow{\mathbb{Z}}}(\lambda) \neq 0$ is satisfied for all $\lambda \in \Lambda_{\overrightarrow{\mathbb{Z}}}$, where

$$
f_{\overrightarrow{\mathbb{Z}}}(\lambda)=\lim _{N_{2}-N_{1} \rightarrow+\infty} \frac{1}{N_{2}-N_{1}} \sum_{N_{1} \leq N_{\leq} \leq N_{2}} \exp (2 \pi i \vec{N} \lambda)
$$

is the structural amplitude (see [1]). From (0.18) it follows that the numbers in the set $\mathbb{Q} \backslash \mathbb{Z}$ do not occur in the spectrum $\Lambda_{\overrightarrow{\mathbb{Z}}}$. A complete description of the spectrum $\Lambda$ is given in Theorem 4.1, where the relation

$$
\Lambda_{\overrightarrow{\mathbb{Z}}}=\mathbb{Z}[\tau]
$$

is obtained. Moreover, it is proved that for any $\lambda=a+b \tau$ in the spectrum $\Lambda_{\overrightarrow{\mathbb{Z}}}$, the amplitude (0.19) is calculated by the formula

$$
f_{\overrightarrow{\mathbb{Z}}}(\lambda)=\frac{\sin (\pi b \tau)}{\pi b \tau} \exp (-3 \pi i b \tau) .
$$

For the one-dimensional quasilattice $\mathcal{F}$ (see $(\overline{0.2})$ ), a description of the spectrum $\Lambda_{\mathcal{F}}$ was given in [14]. It is important to note that the spectra $\Lambda_{\mathcal{F}}$ and $\Lambda_{\overrightarrow{\mathbb{Z}}}$, but not the amplitudes (see Figures 4 and 5), coincide up to the scale multiplier $\nu=1+\tau^{2}$ of nonunit norm, $N_{\mathbb{Q}(\tau) / \mathbb{Q}}(\nu)=5$.

To coordinate the spectra, the renormed values of $\lambda=\nu \lambda_{\mathcal{F}}$ are plotted on the axis $O X$ in Figure 5, where the $\lambda_{\mathcal{F}}$ are taken from the spectrum $\Lambda_{\mathcal{F}}$, and on the axis $O Y$ we have the moduli of the corresponding amplitudes [14]

$$
f_{\mathcal{F}}(\lambda)=\frac{\sin \left(\frac{\pi \tau}{1+\tau^{2}}\left(a+b \tau^{\prime}\right)\right)}{\frac{\pi \tau}{1+\tau^{2}}\left(a+b \tau^{\prime}\right)} \exp \left(-\pi i \frac{1-2 \tau}{1+2 \tau}\left(a+b \tau^{\prime}\right)\right)
$$

where $\tau^{\prime}=-1-\tau$ is conjugate to $\tau$ in the field $\mathbb{Q}(\tau)$.

In J. Lagarias' opinion [16, the sharp diffractional spots allude to the presence of a statistical translational order for far distances between points of a quasilattice. For the Fibonacci quasilattice $\mathcal{F}$, this is a well-known fact (see, e.g., [12]).

On the other hand, the points of the quasilattice $\overrightarrow{\mathbb{Z}}$ also satisfy this condition, because, by the definitions $(0.2)$ and $(0.13)$, the quasilattices $\mathcal{F}$ and $\overrightarrow{\mathbb{Z}}$ have consistent distances between points. A consequence of this is the above-mentioned relation

$$
\nu \cdot \Lambda_{\mathcal{F}}=\Lambda_{\overrightarrow{\mathbb{Z}}}
$$

for their spectra.

The distinction between these quasilattices is that the minimal lattice $\mathcal{L}(\mathcal{F})=\langle 1, \tau\rangle$ that contains $\mathcal{F}$ is generated over $\mathbb{Z}$ by two independent generators 1 and $\tau$, while $\overrightarrow{\mathbb{Z}}$ is contained in the lattice of rational integers

$$
\mathcal{L}(\overrightarrow{\mathbb{Z}})=\mathbb{Z}=\langle 1\rangle .
$$

By formulas (0.20) and (0.21), this property implies the following relations:

$$
\bar{\Lambda}_{\mathcal{F}}^{*}=[0,1], \quad \bar{\Lambda}_{\mathbb{Z}}^{*}=\Lambda_{\overrightarrow{\mathbb{Z}}}^{*} \cup\{0\},
$$

where * denotes the set of values of the moduli of all amplitudes and the bar means the closure of a set, i.e., $\Lambda_{\mathcal{F}}^{*}$ is an everywhere dense subset of the unit interval $[0,1]$, and $\Lambda_{\vec{Z}}^{*}$ is a discrete subset in $[0,1]$ with one limit point 0 .

The Rauzy quasilattice $\mathcal{F}$ (see [2]) is a two-dimensional generalization of the Fibonacci quasilattice $\mathcal{F}$. Its spectrum was studied in $[6]$. 


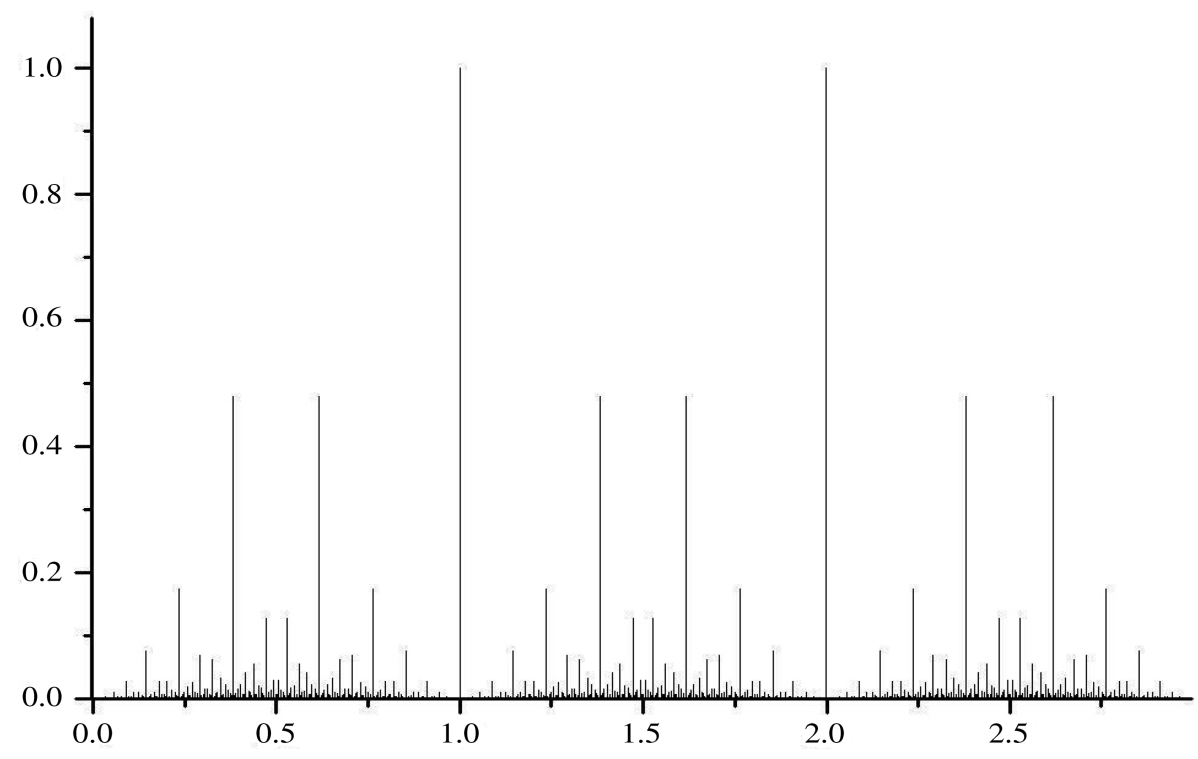

Figure 4. The spectrum of the Fibonacci-even numbers.

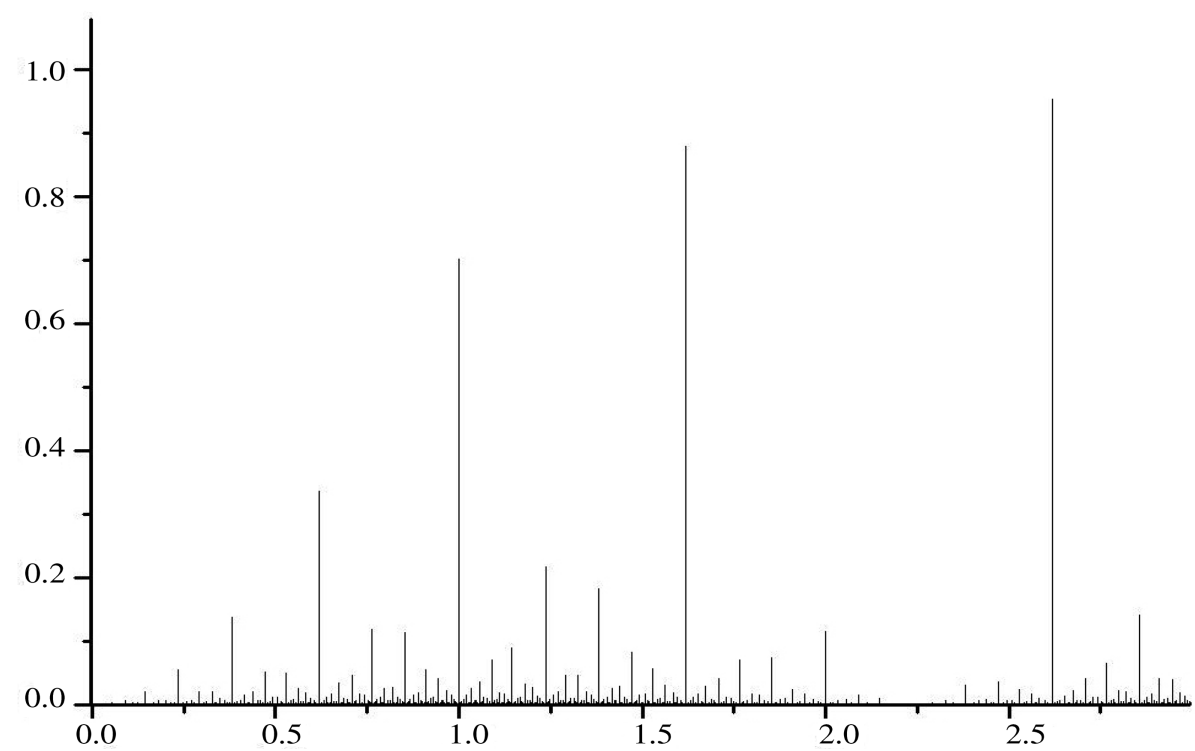

Figure 5. The spectrum $\Lambda_{\mathcal{F}}$ of the one-dimensional Fibonacci quasilattice $\mathcal{F}$.

§1. Binary additive problem

Consider the representations of a natural number $D$ as the sum

$$
\overrightarrow{N_{1}}+\overrightarrow{N_{2}}=D
$$

of two Fibonacci-even numbers $\vec{N}_{i}=F_{1} \circ N_{i}$, where the $N_{i}$ range over the set of numbers $\mathbb{N}=\{0,1,2, \ldots\}$. Applying the $\delta$-map (0.1) to the Diophantine equation (1.1), we obtain 
the congruence

$$
\delta\left(\overrightarrow{N_{1}}\right)+\delta\left(\overrightarrow{N_{2}}\right) \equiv \delta(D) \bmod \mathbb{Z} \widetilde{\tau}
$$

where, by (0.9),

$$
\delta\left(\vec{N}_{i}\right)=\delta\left(F_{1} \circ N_{i}\right)=\delta\left(F_{1}\right) \cdot \delta\left(N_{i}\right)+\left\{\begin{array}{lll}
0 & \text { if } & N_{i} \neq-1, \\
-\widetilde{\tau} & \text { if } & N_{i}=-1,
\end{array}\right.
$$

and

$$
\delta(D) \equiv D \bmod \mathbb{Z} \widetilde{\tau} .
$$

In (1.3) and (1.4), the numbers $N_{i}$ and $D$ may belong to the ring of rational integers $\mathbb{Z}$, and the congruence $x \equiv y \bmod \widetilde{\tau} \mathbb{Z}$ means that the difference $x-y$ belongs to the lattice $\widetilde{\tau} \mathbb{Z}=\{\widetilde{\tau} a ; a \in \mathbb{Z}\}$. Relations (1.2)-(1.4) imply that

$$
-\tau \delta\left(N_{1}\right)-\tau \delta\left(N_{2}\right)=\delta(D)+k \widetilde{\tau},
$$

where $k \in \mathbb{Z}$.

Lemma 1.1. A pair $\left(N_{1}, N_{2}\right)$ is a solution of equation (1.1) if and only if $\left(N_{1}, N_{2}\right)$ is a solution of equation (1.5) for some number $k \in \mathbb{Z}$.

Proof. The "only if" part has already been proved. Conversely, we have $\delta(N)=N-$ $[(N+1) \tau] \widetilde{\tau}$ for any $N \in \mathbb{Z}$, and (1.3), (1.5) imply the relation

$$
\delta\left(\overrightarrow{N_{1}}\right)+\delta\left(\overrightarrow{N_{2}}\right)=\delta(D)+k \widetilde{\tau} .
$$

Consequently, comparing the 1 - and the $\widetilde{\tau}$-component of this relation, we arrive at a system of equations implying (1.1).

Now we find the values of $k \in \mathbb{Z}$ for which equation (1.5) is solvable. Put $x_{i}=\delta\left(N_{i}\right)$ and $d_{k}=-\tilde{\tau} \delta(D)-k \widetilde{\tau}^{2}$, and let

$$
l_{D, k}: x_{2}=-x_{1}+d_{k} E
$$

be lines of level $k$ corresponding to the Diophantine equation (1.1) with free term $D \in \mathbb{N}$.

Lemma 1.2. Suppose that a line $l_{D, k}$ is defined over the field of real numbers $\mathbb{R}$. Then

$$
l_{D, k} \cap J^{2} \neq \varnothing \Leftrightarrow k=0 \text { or } k=1,
$$

with one exception:

$$
l_{D, k} \cap J^{2}=\varnothing \quad \text { if } \delta(D)=-2 \tau^{2}, k=0 .
$$

More precisely, let $l_{D, k} \cap J^{2} \neq \varnothing$. Then

$$
\begin{aligned}
& \text { if } \delta(D) \in\left[-1,-2 \tau^{2}\right), \quad \text { then } \quad k=1 ; \\
& \text { if } \delta(D) \in\left[-2 \tau^{2},-\tau^{2}\right), \quad \text { then } k=0 \quad \text { or } \quad k=1 \text {; } \\
& \text { if } \delta(D) \in\left[-\tau^{2}, \tau\right), \quad \text { then } k=0 .
\end{aligned}
$$

Conversely, if $\delta(D)$ belongs to a half-open interval occurring in (1.7) and the level $k$ is as indicated, then

$$
l_{D, k} \cap J^{2} \neq \varnothing .
$$

Proof. Straightforward calculations.

Now we find the intersection of the lines $l_{D, k}$ with the squared domain $J^{2}$. 
Lemma 1.3. Let

$$
\operatorname{pr}_{D, k}=\operatorname{pr}_{x_{1}}\left(l_{D, k} \cap J^{2}\right)
$$

be the orthogonal projection to the axis $x_{1}$ of the intersection of the line $l_{D, k}$ and the domain $J^{2}$. Then the projection $\operatorname{pr}_{D, k}$ has the form of the following half-open intervals:

$$
\begin{aligned}
& {\left[-1,1+d_{k}\right) \quad \text { if } k=1, \quad \delta(D) \in\left[-1,-2 \tau^{2}\right) ;} \\
& {\left[-1,1+d_{k}\right) \quad \text { if } k=1, \quad \delta(D) \in\left[-2 \tau^{2},-\tau^{2}\right) \text {; }} \\
& {\left[-\tau+d_{k}, \tau\right) \quad \text { if } k=0, \quad \delta(D) \in\left[-2 \tau^{2},-\tau^{2}\right) \text {; }} \\
& {\left[-\tau+d_{k}, \tau\right) \quad \text { if } k=0, \delta(D) \in\left[-\tau^{2}, \tau^{3}\right) \text {; }} \\
& {\left[-1,1+d_{k}\right) \quad \text { if } k=0, \delta(D) \in\left[\tau^{3}, \tau\right) .}
\end{aligned}
$$

Proof. The proof follows from Lemma 1.2 and equation (1.6) for the lines $l_{D, k}$.

Denote

$$
\begin{aligned}
& l_{D}= \begin{cases}l_{D, 0} \cup l_{D, 1} & \text { if } \delta(D) \in\left[-2 \tau^{2},-\tau^{2}\right), \\
l_{D, k} & \text { in other cases; }\end{cases} \\
& \operatorname{pr}_{D}= \begin{cases}\operatorname{pr}_{D, 0} \cup \operatorname{pr}_{D, 1} & \text { if } \delta(D) \in\left[-2 \tau^{2},-\tau^{2}\right), \\
\operatorname{pr}_{D, k} & \text { in other cases. }\end{cases}
\end{aligned}
$$

Then the following chain of equivalences holds true:

$$
\left(N_{1}, N_{2}\right) \in \text { (1.1) } \Leftrightarrow\left(x_{1}, x_{2}\right) \in l_{D} \Leftrightarrow x_{1} \in \mathrm{pr}_{D} .
$$

The first equivalence stems from Lemmas 1.1 and 1.2, and the second is a consequence of Lemma 1.3.

Lemma 1.4. Let $s(D)$ be equal to the number of solutions of the equation $\overrightarrow{N_{1}}+\overrightarrow{N_{2}}=D$, where $N_{i} \in \mathbb{N}$. Then

$$
s(D)=\sharp\left\{N_{1} ; \delta\left(N_{1}\right) \in \operatorname{pr}_{D}, 0 \leq N_{1} \leq\left[\tau D+\tau^{3}\right]\right\},
$$

where $[x]$ is the integral part of $x$.

Proof. If an integer $N$ satisfies

$$
\vec{N}=F_{1} \circ N=N+[(N+1) \tau]=D,
$$

then

$$
\tau D-\tau^{2} \leq N<\tau D+\tau^{3} .
$$

These inequalities and (1.10) imply (1.11).

Now we need a result on the distribution of fractional parts (see, e.g., 7, p. 137]).

The Niederreiter theorem. Suppose a sequence of fractional parts $\{i \alpha\}$, where $i=1$, $2, \ldots, N$, is given, and let $\alpha=\left[q_{0} ; q_{1}, q_{2}, \ldots\right]$ be a continued fraction expansion of an irrational number $\alpha$ with bounded quotients $q_{i} \leq K$ for all $i \geq 1$. Let

$$
A([\beta, \gamma) ; N)=\sharp\{i ;\{i \alpha\} \in[\beta, \gamma), i=1,2, \ldots, N\},
$$

and let

$$
D_{N}=\sup _{0 \leq \beta<\gamma \leq 1}\left|\frac{A([\beta, \gamma) ; N)}{N}-(\gamma-\beta)\right|
$$

be the upper bound of deviations over all half-open intervals for the number of occurrences $A([\beta, \gamma) ; N)$ of the sequence $\{i \alpha\}$ in the half-open interval $[\beta, \gamma)$. Then the following inequality is fulfilled for $D_{N}$ :

$$
N D_{N} \leq 3+\left(\frac{1}{\ln \widetilde{\tau}}+\frac{K}{\ln (K+1)}\right) \ln N .
$$


Let

$$
r_{1}(D)=s(D)-\left(\left[\tau D+\tau^{3}\right]+1\right) \frac{\left|\mathrm{pr}_{D}\right|}{|J|}
$$

be the deviation for the sequence

$$
\delta\left(N_{1}\right) \in J, \quad \text { where } \quad 0 \leq N_{1} \leq\left[\tau D+\tau^{3}\right] .
$$

Then, by Lemma 1.4, $s(D)$ is equal to the number of occurrences of the elements $\delta\left(N_{1}\right)$ in the set $\operatorname{pr}_{D} \subset J$, and $r_{1}(D)$ is the deviation $s(D)$ from the mean value $\left(\left[\tau D+\tau^{3}\right]+1\right) \frac{\left|\mathrm{pr}_{D}\right|}{|J|}$, where $|J|=\widetilde{\tau}$ is the length of the half-open interval $J$ and $\left|\operatorname{pr}_{D}\right|$ is the total length of the half-open intervals from the set $\mathrm{pr}_{D}$ in (1.9).

Lemma 1.5. For the deviation $r_{1}(D)$ in (1.13), we have

$$
\left|r_{1}(D)\right| \leq 3+\left(\frac{1}{\ln \widetilde{\tau}}+\frac{1}{\ln 2}\right) \ln D
$$

for all $D \geq 1$.

Proof. As has already been noted, we may assume that $s(D)$ is equal to the number of occurrences $\delta\left(N_{1}\right)$ in the set $\mathrm{pr}_{D}$, which consists, by (1.9) and Lemma 1.3, of one or two half-open intervals. If we identify the half-open interval $J$ with the circle $C_{\tilde{\tau}}$ of length $\widetilde{\tau}$, then, by (1.9), in the case where $\delta(D) \in\left[-2 \tau^{2},-\tau^{2}\right)$, the set $\operatorname{pr}_{D}$ can be regarded as a single half-open interval in $C_{1}$. For this reason, to estimate the deviation $r(D)$ we can apply inequality (1.12), provided that we additionally use the isomorphism

$$
C_{1} \rightarrow C_{\widetilde{\tau}}: t \mapsto \widetilde{\tau} t .
$$

Next, it suffices to set $\alpha=\tau=[0,1,1, \ldots]$ and $K=1$ in the Niederreiter theorem.

Theorem 1.1. Let $s(D)$ be the number of representations of the natural number $D$ as the sum $\overrightarrow{N_{1}}+\overrightarrow{N_{2}}=D$ of two Fibonacci-even numbers $\overrightarrow{N_{i}}=F_{1} \circ N_{i}$, where $N_{i}=0,1,2, \ldots$ Then the following asymptotic formula holds true for $s(D)$ :

$$
s(D)=c(D) D+r(D),
$$

where (see Figure 3)

$$
c(D)= \begin{cases}-\tau^{3}-\tau \delta(D) & \text { if } \delta(D) \in\left[-1,-2 \tau^{2}\right), \\ \tau^{3} & \text { if } \delta(D) \in\left[-2 \tau^{2},-\tau^{2}\right), \\ 2 \tau^{3}+\tau \delta(D) & \text { if } \delta(D) \in\left[-\tau^{2}, \tau^{3}\right), \\ 2 \tau^{3}-\tau \delta(D) & \text { if } \delta(D) \in\left[\tau^{3}, \tau\right),\end{cases}
$$

and

$$
|r(D)| \leq 5+\left(\frac{1}{\ln \widetilde{\tau}}+\frac{1}{\ln 2}\right) \ln D .
$$

Proof. We use relation (1.13) to write $s(D)$ in the form

$$
\begin{aligned}
s(D) & =\left(\left[\tau D+\tau^{3}\right]+1\right) \frac{\left|\mathrm{pr}_{D}\right|}{|J|}+r_{1}(D) \\
& =\tau^{2} D\left|\operatorname{pr}_{D}\right|+r(D) .
\end{aligned}
$$

Then, by (1.14), we obtain inequality (1.17) for the remainder $r(D)$. The coefficient $c(D)=\tau^{2}\left|\operatorname{pr}_{D}\right|$ in (1.18) is calculated with the help of Lemma 1.3. 
Remark 1.1. Figure 3 depicts the graph of $c(D)$ as a function of $\delta(D)$. This piecewise linear function also admits the following analytic expansion (S. A. Gritsenko, private communication):

$$
c(D)=\tau^{2}+2 \sum_{m=1}^{\infty}\left(\frac{\sin \pi m \tau}{\pi m}\right)^{2} \cos 2 \pi m\left(-\tau^{4}+D \tau\right),
$$

showing that $c(D)$ can be regarded as a function of the fractional part $\{D \tau\}$, linearly connected with $\delta(D)$ in view of formula (0.1).

Remark 1.2. The problem concerning the least upper bound for the remainder $r(D)$ in formula (1.17) seems to be a considerable challenge. The reason is that the number of rotations of the circle and the length of the half-open interval in which the points in question occur vary simultaneously. To prove inequality (1.17), we have used the universal bound (1.12) over all half-open intervals. We can make the remainder $r(D)$ independent of the half-open interval in another way, by using a result of [22]. In particular, it is possible to prove the boundedness of the remainder $r(D)$ for numbers $D$ of bounded length $k(D)$, which is the number of terms in the expansion of $D$ in the Fibonacci number system (0.6).

\section{$\S 2$. Precise formulas for $s(D)$}

2.1. The case where $\delta(D) \in\left[-2 \tau^{2},-\tau^{2}\right)$.

Theorem 2.1. If $D \geq 1$ and $\delta(D) \in\left[-2 \tau^{2},-\tau^{2}\right)$, where $\delta$ is the map (0.1), then the number of solutions of the equation $\overrightarrow{N_{1}}+\overrightarrow{N_{2}}=D$, where $N_{i}=0,1,2, \ldots$, is equal to

$$
s(D)=\left[D \tau^{3}\right] .
$$

Proof. By Lemmas 1.3 and 1.4, the number $s(D)$ of solutions is equal to the number of occurrences $\delta\left(N_{1}\right)$ in the set $\operatorname{pr}_{D}$, which consists of two nonintersecting half-open intervals,

$$
\operatorname{pr}_{D}=\left[-1,1+d_{1}\right) \sqcup\left[-\tau+d_{0}, \tau\right),
$$

as $N_{1}$ ranges over all numbers from 0 to $\left[\tau D+\tau^{3}\right]$, where $\delta\left(N_{1}\right)$ belongs to the half-open interval $J=[-1, \tau)$ and

$$
\delta\left(N_{1}\right)=N_{1}-\left[\left(N_{1}+1\right) \tau\right] \widetilde{\tau} \equiv N_{1} \bmod \widetilde{\tau} \mathbb{Z} .
$$

We introduce the bijection

$$
J \stackrel{*}{\rightarrow} I=[0,1): \quad x \mapsto x^{*}=(x+1) \tau,
$$

which takes a shift

$$
I \stackrel{S^{*}}{\rightarrow} I: \quad x^{*} \mapsto S^{*}\left(x^{*}\right)=x^{*}+\tau \bmod \mathbb{Z}
$$

of the unit half-open interval $I$ to the corresponding shift

$$
J \stackrel{S}{\rightarrow} J: \quad x \mapsto S(x)=x+1 \bmod \widetilde{\tau} \mathbb{Z}
$$

of the half-open interval $J$. Put

$$
\alpha_{1}=1+d_{1}, \quad \alpha_{2}=-\tau+d_{0} .
$$

Since $d_{k}=-\widetilde{\tau} \delta(D)-k \widetilde{\tau}^{2}$, we obtain

$$
\alpha_{1}=1-\widetilde{\tau} \delta(D)-\widetilde{\tau}^{2}=-\widetilde{\tau} \delta(D)-\widetilde{\tau},
$$

which implies the relation

$$
\alpha_{1}^{*}=-\delta(D)-1+\tau, \quad \text { where } \quad \delta(D) \equiv-[(D+1) \tau] \tau \bmod \mathbb{Z},
$$


or the congruence

$$
\alpha_{1}^{*} \equiv([(D+1) \tau]+1) \tau \bmod \mathbb{Z}
$$

Similarly we obtain

$$
\alpha_{2}=-\tau-\tilde{\tau} \delta(D),
$$

and in this case we have the congruence

$$
\alpha_{2}^{*} \equiv([(D+1) \tau]+2) \tau \bmod \mathbb{Z} .
$$

Denoting

$$
i_{D}=[(D+1) \tau],
$$

we see that the above two congruences can be written in the form

$$
\alpha_{1}^{*} \equiv\left(i_{D}+1\right) \tau \bmod \mathbb{Z}, \quad \alpha_{2}^{*} \equiv\left(i_{D}+2\right) \tau \bmod \mathbb{Z} .
$$

By (2.2), we have

$$
\delta(D)^{*}=D \tau-[(D+1) \tau]+\tau=\{D \tau+\tau\} .
$$

Therefore, $\delta(D)^{*}$ lies in the half-open interval $\left[\tau^{4}, \tau^{2}\right)$, because $\left[-2 \tau^{2},-\tau^{2}\right)^{*}=\left[\tau^{4}, \tau^{2}\right)$.

Thus, for the set $\operatorname{pr}_{D}^{*}$ we have the partition

$$
\operatorname{pr}_{D}^{*}=\left[0, \alpha_{1}\right) \sqcup\left[\alpha_{2}^{*}, 1\right),
$$

where $\alpha_{i}^{*}$ are determined from the congruences (2.6). Consider the characteristic function $1_{\left[\alpha_{1}^{*}, \alpha_{2}^{*}\right)}(x)$ of the half-open interval $\left[\alpha_{1}^{*}, \alpha_{2}^{*}\right)$. As is known, it admits the analytic representation

$$
1_{\left[\alpha_{1}^{*}, \alpha_{2}^{*}\right)}(x)=\left(\alpha_{2}^{*}-\alpha_{1}^{*}\right)+\left(\left\{x-\alpha_{2}^{*}\right\}-\left\{x-\alpha_{1}^{*}\right\}\right) .
$$

In order to obtain the characteristic function $1_{\mathrm{pr}_{D}^{*}}(x)$ of the set $\mathrm{pr}_{D}^{*}$, we use the relation $1_{\operatorname{pr}_{D}^{*}}(x)=1-1_{\left[\alpha_{1}^{*}, \alpha_{2}^{*}\right)}(x)$. This and (2.8) yield the representation

$$
1_{\mathrm{pr}_{D}^{*}}(x)=\left|\operatorname{pr}_{D}^{*}\right|+\left(\left\{x-\alpha_{1}^{*}\right\}-\left\{x-\alpha_{2}^{*}\right\}\right),
$$

whence, in view of the congruences (2.6), we conclude that the measure of the set $\mathrm{pr}_{D}^{*}$ is equal to $\left|\operatorname{pr}_{D}^{*}\right|=1-\left(\alpha_{2}^{*}-\alpha_{1}^{*}\right)=\tau^{2}$. Thus, (2.9) leads to the formula

$$
1_{\mathrm{pr}_{D}^{*}}(x)=\tau^{2}+\left(\left\{x-\left(i_{D}+1\right) \tau\right\}-\left\{x-\left(i_{D}+2\right) \tau\right\}\right) .
$$

Now, in view of Lemma 1.4, the number $s(D)$ of solutions of the equation $\overrightarrow{N_{1}}+\overrightarrow{N_{2}}=D$ can be written with the help of the characteristic function in the form

$$
s(D)=\sum_{0 \leq N_{1} \leq\left[\tau D+\tau^{3}\right]} 1_{\operatorname{pr}_{D}^{*}}\left(\delta\left(N_{1}\right)\right) .
$$

Since $\delta\left(N_{1}\right) \equiv\left(N_{1}+1\right) \tau \bmod \mathbb{Z}$ by (2.7), we write (2.11) in the form

$$
s(D)=\sum_{1 \leq N_{1} \leq\left[\tau D+\tau^{3}\right]+1} 1_{\operatorname{pr}_{D}^{*}}(i \tau) .
$$

We have $\left[\tau D+\tau^{3}\right]=\left[(\tau D+\tau)-\tau^{2}\right]$, where $\{\tau D+\tau\}$ lies in the half-open interval $\left[\tau^{4}, \tau^{2}\right)$. Hence,

$$
\left[\tau D+\tau^{3}\right]=[\tau D+\tau]-1=i_{D} .
$$

Therefore, the sum (2.12) can be represented as

$$
s(D)=\sum_{1 \leq i \leq i_{D}} 1_{\mathrm{pr}_{D}^{*}}(i \tau)=i_{D} \tau^{2}+\Sigma_{1}-\Sigma_{2},
$$


where, by (2.10) and (2.6),

$$
\begin{aligned}
& \Sigma_{1}=\sum_{1 \leq i \leq i_{D}}\left\{i \tau-\left(i_{D}+1\right) \tau\right\}, \\
& \Sigma_{2}=\sum_{1 \leq i \leq i_{D}}\left\{i \tau-\left(i_{D}+2\right) \tau\right\} .
\end{aligned}
$$

The expressions (2.15) for the sums $\Sigma_{1}$ and $\Sigma_{2}$ make it possible to find their difference:

$$
\Sigma_{1}-\Sigma_{2}=\{-\tau\}-\left\{-\left(i_{D}+1\right) \tau\right\},
$$

and thus, by (2.14), for $s(D)$ we have

$$
s(D)=\left[-\left(i_{D}+1\right) \tau^{2}\right]=\left[([(D+1) \tau]+1) \tau^{2}\right] .
$$

Consider the following auxiliary function:

$$
R(D)=\left[([(D+1) \tau]+1) \tau^{2}\right]-\left[D \tau^{3}\right] .
$$

Direct inspection shows that the function $R(D)$ defined in this way can take only two values, 0 or 1:

$$
R(D)= \begin{cases}0 & \text { if } \delta(D) \in\left[-2 \tau^{2},-\tau^{2}\right) \cup[0, \tau), \\ 1 & \text { otherwise }\end{cases}
$$

Formulas (2.16) and (2.1) yield the required formula (2.1).

2.2. The case of $D=F_{n}$. First, we note that

$$
\delta\left(F_{n}\right)=(-\tau)^{n} \quad \text { for } \quad n \geq 1,
$$

where the $F_{n}$ are the Fibonacci numbers (0.5). Indeed, for Fibonacci numbers, formula (0.7) for o-multiplication takes the form $F_{1} \circ F_{n-1}=F_{n}$, and, by the definition (0.1) of the $\delta$-map, we have $\delta\left(F_{1} \circ F_{n-1}\right)=\delta\left(F_{n}\right)$, where $\delta\left(F_{1}\right)=-\tau$. This implies (2.18), yielding

$$
\delta\left(F_{n}\right) \in\left[-\tau^{2}, \tau^{3}\right) \quad \text { for } \quad n \geq 3 .
$$

By Lemma 1.3, we have

$$
\operatorname{pr}_{D}=\left[-\tau+d_{0}, \tau\right)=[-\tau-\widetilde{\tau} \delta(D), \tau)=\left[\alpha_{1}, \alpha_{2}\right),
$$

whence

$$
\operatorname{pr}_{D}^{*}=\left[\alpha_{1}^{*}, \alpha_{2}^{*}\right)=\left[\left(i_{D}+2\right) \tau, 1\right),
$$

where

$$
i_{D}^{*}=[(D+1) \tau]=\left[F_{n} \tau+\tau\right]=F_{n-1} .
$$

Thus, we obtain an explicit form of the characteristic function of the half-open interval $\operatorname{pr}_{D}^{*}$, namely,

$$
1_{\operatorname{pr}_{D}^{*}}(x)=\left(1-\left\{\left(i_{D}+2\right) \tau\right\}\right)+\{x\}-\left\{x-\left(i_{D}+2\right) \tau\right\},
$$

where

$$
\left\{\left(i_{D}+2\right) \tau\right\}=\left\{F_{n-1} \tau+2 \tau\right\}=(-1)^{n-1} \tau^{n}+\tau^{3} .
$$

Similarly, we write (2.14) in the form

$$
s(D)=\sum_{1 \leq N_{1} \leq\left[\tau D+\tau^{3}\right]+1} 1_{\operatorname{pr}_{D}^{*}}(i \tau) .
$$

Since $\delta^{*}(D) \equiv D \tau+\tau \bmod \mathbb{Z}$, it follows that $\delta^{*}(D)$ belongs to the half-open interval $\left[\tau^{2}, \tau+\tau^{4}\right)$, and thus, by (2.19) and (2.21), we have

$$
\left[\tau D+\tau^{3}\right]=[\tau D+\tau]=i_{D}=F_{n-1} .
$$


Using (2.22) and (2.24), from formula (2.23) we obtain

$$
s\left(F_{n}\right)=\left(1+(-1)^{n} \tau^{n}-\tau^{3}\right)\left(F_{n-1}+1\right)+\sigma\left(F_{n}+F_{1}\right)
$$

for $n \geq 3$, where

$$
\sigma(D)=\sum_{1 \leq i \leq D}(2\{i \tau\}-1)
$$

Using the Brown formula [11, we can calculate the sum $\sigma(D)$ explicitly:

$$
\sigma\left(F_{n}+F_{1}\right)=\alpha_{n}+\varrho_{n},
$$

where

$$
\alpha_{n}=\left\{\begin{array}{lll}
2 \tau-\frac{1}{\sqrt{5}} & \text { if } & n \text { is even } \\
2 \tau^{2}+\frac{1}{\sqrt{5}} & \text { if } & n \text { is odd }
\end{array}\right.
$$

and

$$
\varrho_{n}=-3(-1)^{n} \tau^{n}+\frac{\tau^{2 n}}{\sqrt{5}}
$$

Theorem 2.2. The number of solutions of the equation $\overrightarrow{N_{1}}+\overrightarrow{N_{2}}=F_{n}$, where $N_{i} \geq 0$, is equal to

$$
s\left(F_{n}\right)=F_{n-1}-F_{n-4}+2 e(n),
$$

and $e(n)=1$ or 0 for $n$ even or odd, respectively.

Proof. We write (2.25) in the form

$$
s\left(F_{n}\right)=\left[\left(1+(-1)^{n} \tau^{n}-\tau^{3}\right)\left(F_{n-1}+1\right)\right]+a_{n},
$$

where

$$
a_{n}=\left\{\left(1+(-1)^{n} \tau^{n}-\tau^{3}\right)\left(F_{n-1}+1\right)\right\}+\sigma\left(F_{n}+f_{1}\right)
$$

is a rational integer. From (2.26)-(2.28) it readily follows that $a_{n}=0$ or 1 . To calculate $a_{n}$ more precisely, we use the explicit form of the integral part in formula (2.30),

$$
\left[\left(1+(-1)^{n} \tau^{n}-\tau^{3}\right)\left(F_{n-1}+1\right)\right]=F_{n-1}-F_{n-4}+e_{n},
$$

where

$$
e_{n}= \begin{cases}1 & \text { if } n \text { is even, } \\ 0 & \text { if } n>3 \text { is odd } \\ -1 & \text { if } n=3\end{cases}
$$

and the relation for the corresponding fractional part:

$$
\left\{\left(1+(-1)^{n} \tau^{n}-\tau^{3}\right)\left(F_{n-1}+1\right)\right\}=1-\tau^{3}+\frac{(-1)^{n}}{\sqrt{5}}-e_{n}+3(-1)^{n} \tau^{n}-\frac{\tau^{2 n}}{\sqrt{5}} .
$$

By (2.33) and (2.26) -(2.28), the following formula is valid for $a_{n}$ :

$$
a_{n}= \begin{cases}1 & \text { if } n \text { is even } \\ 0 & \text { if } n>3 \text { is odd } \\ 1 & \text { if } n=3\end{cases}
$$

Since $a_{n}+e_{n}=2 e(n)$, the above formula and (2.30), (2.32) imply formula (2.29). 
Remark 2.1. The method of proving Theorem 2.2 can also be applied to the proof of explicit formulas for the number of solutions of the equation $\overrightarrow{N_{1}}+\overrightarrow{N_{2}}=D$ in the case where

$$
D=F_{n+n_{1}}+\cdots+F_{n+n_{k}}
$$

here the indices $0 \leq n_{1}<\cdots<n_{k}$ are fixed, $n_{i}<n_{i+1}-1$, and the index $n$ is an arbitrary natural number. In particular, the above-mentioned formulas for numbers $D$ of the form (2.34) imply the boundedness of the remainder $r(D)$ in formula (1.17). Thus, we obtain (see Remark 1.2) an alternative approach to 22 for estimating the remainder $r(D)$ for numbers $D$ that have bounded length $k=k(D)$ in the Fibonacci number system (0.6).

\section{§3. UNIFORM DISTRIBUTION OVER PROGRESSIONS}

Let $d>1$ and $r \geq 0$ be rational integers. We consider the problem concerning the occurrence of Fibonacci-even numbers $\overrightarrow{N_{1}}$ in the arithmetic progression

$$
\mathcal{P}(d, r)=\left\{r+d N_{2} ; N_{2} \in \mathbb{N}\right\} .
$$

For this, we introduce the Diophantine equation

$$
\overrightarrow{N_{1}}-d N_{2}=r
$$

and find the number of its solutions $N_{1}, N_{2} \in \mathbb{N}$ satisfying $0 \leq N_{1} \leq X$ as $X \rightarrow+\infty$. Thereby, we shall find the number of Fibonacci-even numbers $\overrightarrow{N_{1}}$ that occur in the progression $\mathcal{P}(d, r)$.

Applying the map $\delta$ (see (10.1)) to equation (3.1), we arrive at the congruence

$$
\delta\left(\overrightarrow{N_{1}}-d N_{2}\right)=\delta(r) \equiv r \bmod \widetilde{\tau} \mathbb{Z},
$$

and

$$
\delta\left(\overrightarrow{N_{1}}-d N_{2}\right) \equiv-\tau \delta\left(N_{1}\right)-d \delta\left(N_{2}\right) \bmod \widetilde{\tau} \mathbb{Z}
$$

Hence,

$$
-\tau \delta\left(N_{1}\right)-d \delta\left(N_{2}\right)=r+k \widetilde{\tau},
$$

where $k$ is a rational integer.

Lemma 3.1. Let $N_{1}$ and $N_{2}$ be arbitrary rational integers. Then $\left(N_{1}, N_{2}\right)$ is a solution of the Diophantine equation (3.1) if and only if $\left(N_{1}, N_{2}\right)$ is a solution of equation (3.2) with some $k \in \mathbb{Z}$.

Proof. Only the "if" part needs a proof. Let $\left(N_{1}, N_{2}\right)$ be a solution of (3.2). Then, using formulas (1.3) and (1.4), from (3.2) we deduce that $\left(N_{1}, N_{2}\right)$ satisfies the system of equations

$$
\begin{aligned}
\overrightarrow{N_{1}}-d N_{2} & =r, \\
{\left[\left(\overrightarrow{N_{1}}+1\right) \tau\right]-\left[\left(N_{2}+1\right) \tau\right] } & =k .
\end{aligned}
$$

Thus, $\left(N_{1}, N_{2}\right)$ is a solution of (3.1).

We introduce the variables $x_{i}=\delta\left(N_{i}\right)$ and consider the lines

$$
l_{d, r, k}: \quad x_{1}=-\widetilde{\tau} d x_{2}-r \widetilde{\tau}-k \widetilde{\tau} .
$$

The restrictions $x_{i} \in J$ on the variables $x_{i}$ imply that the condition

$$
\bar{l}_{d, r, k} \cap J^{2} \neq \varnothing,
$$


where $\bar{l}_{d, r, k}$ is the closure of the line $l_{d, r, k}$, is satisfied if and only if the level $k$ belongs to the set

$$
K_{d, r}=\left(-\tau\left(\tau^{2}-\tau d+r\right), \tau(\tau+d-r)\right] \cap \mathbb{Z} .
$$

A straightforward calculation yields the following statement.

Lemma 3.2. Let

$$
\operatorname{pr}_{d, r, k}=\operatorname{pr}_{x_{2}}\left(\bar{l}_{d, r, k} \cap J^{2}\right) \subset J
$$

be projections to the ordinate axis. Then for $k \in K_{d, r}$, the projections $\mathrm{pr}_{d, r, k}$ are disjoint, and if we identify the half-open interval $J$ with the circle, then the set $\bigsqcup_{k \in K_{d, r}} \operatorname{pr}_{d, r, k}$ consists of $d$ nonintersecting half-open intervals, each of length $\frac{1}{d}$.

Let $x_{i}=\delta\left(N_{i}\right)$, where $N_{i} \in \mathbb{Z}$. Then

$$
\left(x_{1}, x_{2}\right) \in l_{d, r, k} \quad \Leftrightarrow \quad x_{2}=\delta\left(N_{2}\right) \in \operatorname{pr}_{d, r, k} .
$$

If $0 \leq N_{1} \leq X$, then equation (3.1) implies that $0 \leq N_{2} \leq N_{2}(X)$, where

$$
N_{2}(X)=\frac{\widetilde{\tau}}{d} X+c_{d, r}(X)
$$

and the remainder $\left|c_{d, r}(X)\right|$ is bounded by a quantity independent of $d, r$, and $X$. Applying the Niederreiter theorem, we obtain

$$
\begin{aligned}
& \sharp\left\{N_{2} ; \delta\left(N_{2}\right) \in \mathrm{pr}_{d, r, k}, 0 \leq N_{2} \leq N_{2}(X)\right\} \\
& =\frac{\left|\mathrm{pr}_{d, r, k}\right|}{|J|} N_{2}(X)+c_{d, r, k}^{\prime}(X) \ln N_{2}(X),
\end{aligned}
$$

where $\left|c_{d, r, k}^{\prime}(X)\right|$ is bounded by an absolute constant and the value

$$
\frac{\left|\mathrm{pr}_{d, r, k}\right|}{|J|}=\frac{\tau}{d}
$$

of the normed length belongs to the quadratic field $\mathbb{Q}(\tau)$. If, in accordance with Lemma 3.2 , the half-open intervals $\mathrm{pr}_{d, r, k_{1}}$ and $\mathrm{pr}_{d, r, k_{2}}$ are such that $k_{1}$ and $k_{2}$ are, respectively, the smallest and the largest numbers in the set of admissible parameters $K_{d, r}$, then we arrive at the same relation

$$
\frac{\left|\operatorname{pr}_{d, r, k_{1}}\right|+\left|\operatorname{pr}_{d, r, k_{2}}\right|}{|J|}=\frac{\tau}{d}
$$

Moreover, in formula (3.6) the half-open interval $\mathrm{pr}_{d, r, k}$ is replaced by the union $\operatorname{pr}_{d, r, k_{1}} \sqcup$ $\mathrm{pr}_{d, r, k_{2}}$ of two nonintersecting half-open intervals.

Formulas (3.6)-(3.8) and Lemma 3.2 yield the asymptotic formula

$$
\sharp\left\{N_{2} ; \delta\left(N_{2}\right) \in \bigsqcup_{k \in K_{d, r}} \operatorname{pr}_{d, r, k}, 0 \leq N_{2} \leq N_{2}(X)\right\}=\frac{X}{d}+c(d) \ln X,
$$

where $c(d)=O(d \ln d)$ and the constant in $O$ is independent of $X, d$, and $r$.

Theorem 3.1. Let $l_{F_{1}}(d, r, X)$ be the number of Fibonacci-even numbers $\vec{N}=F_{1} \circ N$, $0 \leq N \leq X$, that satisfy the congruence $\vec{N} \equiv r \bmod d$, where $d$ and $r$ are arbitrary rational integers and $d>1$. Then the following asymptotic formula is valid for $l_{F_{1}}(d, r, X)$ :

$$
l_{F_{1}}(d, r, X)=\frac{X}{d}+c(d) \ln X
$$

as $X \rightarrow+\infty$, where $c(d)$ is as in (3.9). 
Proof. By definition, $l_{F_{1}}(d, r, X)$ is the number of solutions of the equation $\vec{N}-d N_{2}=r$, where $0 \leq N_{1} \leq X$. Then, by Lemmas 3.1 and $3.2, l_{F_{1}}(d, r, X)$ is equal to the number of $N_{2}$ 's that satisfy $0 \leq N_{2} \leq N_{2}(X)$ and $\delta\left(N_{2}\right)$ occurs in the set $\bigsqcup_{k \in K_{d, r}} \operatorname{pr}_{d, r, k}$. Thus, the asymptotic formula (3.9) implies (3.10).

Remark 3.1. Lemma 3.2 and the results of [10] show that there exists an infinite sequence of numbers $X_{i}, X_{i} \rightarrow+\infty$ as $i \rightarrow+\infty$, for which the remainder in formula (3.10) satisfies the inequality

$$
\left|l_{F_{1}}\left(d, r, X_{i}\right)-\frac{X_{i}}{d}\right|>c \ln X_{i},
$$

where the difference $d>1$ of progressions is fixed, and $c>0$ is a constant depending only on $d$. Thus, the bound for the remainder contained in formula 3.10 is order-sharp. Estimation of the remainder from below as the difference $d$ of the arithmetic progressions grows is an open problem.

Remark 3.2. The method of this section makes it possible to study the distribution over progressions of the numbers $N_{A}=A \circ N$, where $A \neq 0$ is an arbitrary rational integer and $\circ$ is the circular Fibonacci multiplication (0.8). For example, if one of the Fibonacci numbers $F_{n}$ is taken for the role of $A$, then, much as this has been done for (3.10), we can prove the asymptotic formula

$$
l_{A}(d, r, X)=\frac{X}{d}+c_{A}(d) \ln X,
$$

where $c_{A}(d)=O_{A}(d \ln d)$ and the constant in $O_{A}$ depends only on $A=F_{n}$. If $A$ is arbitrary, it is necessary to require that the parameters $A, d$, and $r$ be consistent so as to ensure the solvability of the congruence

$$
A \circ N \equiv r \bmod d .
$$

We observe that, for $A=F_{n}$, its $\delta$-image $\delta(A)=(-\tau)^{n}$ is a unit of the ring $\mathbb{Z}[\tau]$ of integers of the quadratic field $\mathbb{Q}(\tau)$. Consequently, in this case, $A, d$, and $r$ are consistent automatically.

\section{§4. Spectrum of FibonacCi-even numbers}

4.1. Torus windings. For a sequence (see (0.13)

$$
a_{N}=\vec{N}=N+[(N+1) \tau]
$$

where $N \in \mathbb{Z}$, we consider the Fourier transform (for rigorous definitions, see [13, 16, 20, 21])

$$
F_{\overrightarrow{\mathbb{Z}}}(x)=\sum_{\lambda \in \Lambda} f_{\overrightarrow{\mathbb{Z}}}(\lambda) \delta(x-\lambda),
$$

where

$$
f_{\overrightarrow{\mathbb{Z}}}(\lambda)=\lim _{N_{2}-N_{1} \rightarrow+\infty} \frac{1}{N_{2}-N_{1}} \sum_{N_{1} \leq N \leq N_{2}} e\left(a_{N} \lambda\right)
$$

is the structural amplitude; here we use the notation $e(x)=\exp (2 \pi i x)$, and the sum (4.1) contains the Dirac $\delta$-function

$$
\delta(x)=\left\{\begin{array}{lll}
1 & \text { if } & x=0 \\
0 & \text { if } & x \neq 0
\end{array}\right.
$$

We write the product $a_{N} \lambda$ in the form

$$
a_{N} \lambda=N \lambda \widetilde{\tau}-\{(N+1) \tau\} \lambda+\lambda \tau
$$


and define the vector

$$
\alpha=\alpha_{\lambda}=\left(\alpha_{1}, \alpha_{2}\right)=(\lambda \widetilde{\tau}, \tau) .
$$

On the torus $\mathbb{T}^{2}=\mathbb{R}^{2} / \mathbb{Z}^{2}$, we consider the points

$$
x_{N}=N \alpha+x_{0} \bmod \mathbb{Z}^{2},
$$

where $x_{0}=(0, \tau)$ and $N \in \mathbb{Z}$. This set forms the orbit

$$
\operatorname{Orb}\left(\alpha, x_{0}\right)=\left\{S_{\alpha}^{N}\left(x_{0}\right) ; N \in \mathbb{Z}\right\}
$$

obtained from the initial point $x_{0}$ with the help of the following shift of the torus:

$$
\mathbb{T}^{2} \stackrel{S_{\alpha}}{\longrightarrow} \mathbb{T}^{2}: x \mapsto S_{\alpha}(x)=x+\alpha \bmod \mathbb{Z}^{2} .
$$

Lemma 4.1. The points of the orbit

$$
\operatorname{Orb}\left(\alpha, x_{0}\right)=\operatorname{Orb}(\alpha)+x_{0} \bmod \mathbb{Z}^{2},
$$

where $\operatorname{Orb}(\alpha)=\operatorname{Orb}(\alpha, 0)$, are uniformly distributed on its closure

$$
\overline{\operatorname{Orb}}\left(\alpha, x_{0}\right)=\overline{\operatorname{Orb}}(\alpha)+x_{0} \bmod \mathbb{Z}^{2} .
$$

Proof. This follows from the irrationality of the golden section $\tau$.

Using the shift $S_{\alpha}$ and (4.3), we can represent the amplitude $f_{\overrightarrow{\mathbb{Z}}}(\lambda)$ in the form

$$
f_{\overrightarrow{\mathbb{Z}}}(\lambda)=\lim _{N_{2}-N_{1} \rightarrow+\infty} \frac{1}{N_{2}-N_{1}} \sum_{N_{1} \leq N \leq N_{2}} \mathbf{e}\left(S_{\alpha}^{N}\left(x_{0}\right)\right),
$$

where

$$
\mathbf{e}(y)=e\left(y_{1}-y_{2} \lambda+\lambda \tau\right)
$$

for an arbitrary vector $y=\left(y_{1}, y_{2}\right)$ in $\mathbb{R}^{2}$.

Case 1. If $\lambda$ does not belong to the quadratic field $\mathbb{Q}(\tau)$, then the numbers $1, \alpha_{1}, \alpha_{2}$ are linearly independent over $\mathbb{Z}$. This implies that

$$
\overline{\operatorname{Orb}}\left(\alpha, x_{0}\right)=\mathbb{T}^{2},
$$

and moreover, by the Weil theorem (see, e.g., [7), the set of points of $\operatorname{Orb}\left(\alpha, x_{0}\right)$ is uniformly distributed on the torus $\mathbb{T}^{2}$. The above-mentioned properties make it possible to calculate the amplitude (4.8) straightforwardly:

$$
f_{\overrightarrow{\mathbb{Z}}}(\lambda)=\int_{0}^{1} \int_{0}^{1} e\left(y_{1}-y_{2} \lambda+\lambda \tau\right) d y_{1} d y_{2}=0 .
$$

Case 2. Now, let $\lambda \in \mathbb{Q}(\tau)$. This case calls for a more sophisticated treatment, because now the numbers $1, \alpha_{1}, \alpha_{2}$ are linearly dependent over $\mathbb{Z}$. For this reason, the closure of the orbit $\operatorname{Orb}(\alpha)$ is split

$$
\overline{\operatorname{Orb}}(\alpha)=\bigsqcup_{i} W\left(v, s_{i}\right)
$$

into a disjoint union of torus windings

$$
W\left(v, s_{i}\right)=\left\{S_{v}^{t}\left(s_{i}\right) ; t \in \mathbb{R}\right\} \subset \mathbb{T}^{2},
$$

where

$$
\mathbb{T}^{2} \stackrel{S_{v}^{t}}{\longrightarrow} \mathbb{T}^{2}: x \mapsto S_{v}^{t}(x)=x+t v \bmod \mathbb{Z}^{2}
$$

is a flow on the torus $\mathbb{T}^{2}$ that is generated by the direction vector $v=\left(v_{1}, v_{2}\right)$. The decomposition (4.11) is a union of finitely many periodic windings $W\left(v, s_{i}\right)$ obtained by the parallel shifts

$$
W\left(v, s_{i}\right)=W(v)+s_{i} \bmod \mathbb{Z}^{2}
$$

of the winding $W(v)=W(v, 0)$ issuing out of the origin. 
Each periodic winding $W\left(v, s_{i}\right)$ admits a canonical representation

$$
W\left(v, s_{i}\right)=\left\{S_{v}^{t}\left(s_{i}\right) ; t \in I\right\},
$$

where $I=[0,1)$ and the direction vector $v$ has the form

$$
v=\left(v_{1}, v_{2}\right) \in \mathbb{Z}^{2} \backslash\{(0,0)\}, \quad \text { where } \quad \text { G.C.D. }\left(v_{1}, v_{2}\right)=1 .
$$

Relation (4.14) shows that the map

$$
I \stackrel{S_{v}}{\longrightarrow} W\left(v, s_{i}\right): t \mapsto x=S_{v}^{t}\left(s_{i}\right)
$$

is a bijection.

Since $\lambda \in \mathbb{Q}(\tau)$ by assumption, $\lambda$ can be uniquely represented in the form

$$
\lambda=\frac{a+b \tau}{c}, \quad a, b, c \in \mathbb{Z}, \quad c>0,
$$

and the numbers $a, b, c$ have no nontrivial common divisor. Moreover, let

$$
a=d a_{1}, \quad c=d c_{1}, \quad \text { where } \quad d=\text { G.C.D. }(a, c) .
$$

For such a $\lambda$, the direction vector (4.4) has the following canonical form:

$$
\alpha=\alpha_{\lambda}=\left(\frac{(a+b)+a \tau}{c}, \tau\right) .
$$

Proposition 4.1. If the direction vector $\alpha$ is of the form (4.4), then the closure of the orbit $\operatorname{Orb}(\alpha)=\operatorname{Orb}(\alpha, 0)$ is split,

$$
\overline{\operatorname{Orb}}(\alpha)=\bigsqcup_{i \bmod d} W\left(v, s_{i}\right),
$$

into nonintersecting torus windings $W\left(v, s_{i}\right)$ with the direction vector $v=\left(a_{1}, c_{1}\right)$ and the initial points $s_{i}=\left(\frac{i}{c}, 0\right)$.

Proof. To find the direction vector $v$, we note that, by (4.5) and (4.18), the orbit $\operatorname{Orb}(\alpha)$ is formed by the points

$$
x_{N}=\left(\frac{a+b}{c} N+\frac{a}{c} N \tau, N \tau\right) \bmod \mathbb{Z}^{2},
$$

where $N \in \mathbb{Z}$. We take $N=c F_{m} N_{1}$; then

$$
x_{N} \equiv d N_{1}\left(a_{1} F_{m} \tau, c_{1} F_{m} \tau\right) \bmod \mathbb{Z}^{2},
$$

where the vector $\left(a_{1} F_{m} \tau, c_{1} F_{m} \tau\right)$ can be made arbitrarily small mod $\mathbb{Z}^{2}$ if the index $m$ is taken sufficiently large. The congruence (4.21) implies that all windings in the closure of the orbit $\overline{\operatorname{Orb}}(\alpha)$ have one and the same direction vector $v=\left(a_{1}, c_{1}\right)$, because, by condition (4.17), the integers $a_{1}$ and $c_{1}$ are coprime.

We introduce the subset

$$
\operatorname{Orb}_{\mathbb{A}}\left(\alpha^{\prime}, 0\right)=\left\{x_{N}^{\prime}=N \alpha^{\prime} \bmod \mathbb{Z}^{2} ; N \in \mathbb{A}\right\}
$$

in the orbit $\operatorname{Orb}\left(\alpha^{\prime}, 0\right)$, where

$$
\alpha^{\prime}=\left(\alpha_{1}, \alpha_{2}\right)=\left(\frac{a}{c} \tau, \tau\right)
$$

and $\mathbb{A}$ is a subset of $\mathbb{Z}$. Then it is easily seen that the following properties are fulfilled:

$$
\operatorname{Orb}\left(\alpha^{\prime}, 0\right) \subset W(v, 0), \quad \overline{\operatorname{Orb}}\left(\alpha^{\prime}, 0\right)=W(v, 0),
$$

where $v=\left(a_{1}, c_{1}\right)$; moreover, we have

$$
\overline{\operatorname{Orb}}_{\mathbb{A}}\left(\alpha^{\prime}, 0\right)=\overline{\operatorname{Orb}}\left(\alpha^{\prime}, 0\right)
$$

for any infinite subset $\mathbb{A}$ in $\mathbb{Z}$. The properties mentioned above imply the relation

$$
\overline{\operatorname{Orb}}_{\mathbb{A}}\left(\alpha^{\prime}, 0\right)=W(v, 0) .
$$


The winding $W(v, 0) \subset \mathbb{T}^{2}$ intersects the axis $x_{1}$ at the points

$$
\frac{j}{c_{1}}, \quad \text { where } j \text { is reduced } \bmod c_{1} \text {. }
$$

As $\mathbb{A} \subset \mathbb{Z}$ we take the subsets

$$
\mathbb{A}_{i}=\{N ;(a+b) N \equiv i \bmod c, N \in \mathbb{Z}\} .
$$

Then from (4.21) we obtain the decomposition

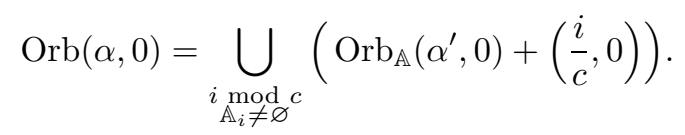

Passing to closures, we see that

$$
\overline{\operatorname{Orb}}(\alpha, 0)=\bigcup_{\substack{i \\ \mathbb{A}_{i} \neq \varnothing}}\left(\overline{\operatorname{Orb}_{\mathbb{A}}}\left(\alpha^{\prime}, 0\right)+\left(\frac{i}{c}, 0\right)\right)=\bigcup_{\substack{i \\ \mathbb{A}_{i} \neq \varnothing}} W\left(v,\left(\frac{i}{c}, 0\right)\right) .
$$

Then for the closure of the orbit $\overline{\operatorname{Orb}}(\alpha, 0)$ we obtain the following partition into torus windings:

$$
\overline{\operatorname{Orb}}(\alpha, 0)=\bigsqcup_{t \in \frac{a+b}{c} \mathbb{Z} / \frac{1}{c_{1}} \mathbb{Z}} W(v,(t, 0)),
$$

where $r \mathbb{Z}=\{r N ; N \in \mathbb{Z}\}$ is the cyclic subgroup with generating element $r \in \mathbb{Q}$ and $t$ ranges over all representatives from the quotient of the group $\frac{a+b}{c} \mathbb{Z}$ by the subgroup

$$
\frac{1}{c_{1}} \mathbb{Z} \subset \frac{a+b}{c} \mathbb{Z} .
$$

Next we apply the localization method. Let $c=p^{\alpha}$, where $p$ is a prime number.

(1) If $p \mid a$, then $p \nmid b$. Hence, we have $p \nmid(a+b)$, whence

$$
\frac{a+b}{c} \mathbb{Z}=\frac{1}{c} \mathbb{Z}=\bigsqcup_{i \bmod d}\left(\frac{i}{c_{1} d}+\frac{i}{c_{1}} \mathbb{Z}\right)
$$

is a disjoint partition into cosets.

(2) If $p \nmid a$, then $c_{1}=c$, so that

$$
\frac{a+b}{c} \mathbb{Z}=\frac{1}{c_{1}} \mathbb{Z} .
$$

Looking over all prime divisors $p \mid c$, from (4.28) and (4.29) we deduce the validity of (4.28) for any integer $c \neq 0$, and, with it, of the inclusion (4.27).

This and (4.26) imply (4.19).

4.2. Spectrum. Proposition 4.1, relation (4.2), and Lemma 4.1 show that the following representation for the amplitude $f_{\overrightarrow{\mathbb{Z}}}(\lambda)$ is valid:

$$
f_{\overrightarrow{\mathbb{Z}}}(\lambda)=e(\lambda \tau) \frac{1}{d} \sum_{i \bmod d} e\left(\frac{i}{c}\right) I_{1}\left(\lambda, a_{1}, c_{1}\right),
$$

where

$$
I_{1}\left(\lambda, a_{1}, c_{1}\right)=\int_{0}^{1} e\left(a_{1} t-\left\{c_{1} t+\tau\right\} \lambda\right) d t .
$$


We divide the integration interval $[0,1]$ into intervals of the form $\left[\frac{j}{c_{1}}, \frac{j+1}{c_{1}}\right]$ and make the change of variables $t=\frac{j}{c_{1}}+\frac{\Delta}{c_{1}}$. After that, the integral (4.31) expands into a sum:

$$
\begin{aligned}
I_{1}\left(\lambda, a_{1}, c_{1}\right) & =\frac{1}{c_{1}} \sum_{j \bmod c_{1}} \int_{0}^{1} e\left(\frac{a_{1} j}{c_{1}}+\frac{a_{1} \Delta}{c_{1}}-\{\Delta+\tau\} \lambda\right) d \Delta \\
& =\frac{1}{c_{1}} \sum_{j \bmod c_{1}} e\left(\frac{a_{1} j}{c_{1}}\right) I_{2}\left(\lambda, a_{1}, c_{1}\right),
\end{aligned}
$$

where

$$
I_{2}\left(\lambda, a_{1}, c_{1}\right)=\int_{0}^{1} e\left(\frac{a_{1} \Delta}{c_{1}}-\{\Delta+\tau\} \lambda\right) d \Delta .
$$

By (4.30) and (4.32), we have

$$
f_{\overrightarrow{\mathbb{Z}}}(\lambda)=e(\lambda \tau) I_{2}\left(\lambda, a_{1}, c_{1}\right) \sigma(c),
$$

where

$$
\sigma(c)=\frac{1}{c} \sum_{i \bmod d} \sum_{j \bmod c_{1}} e\left(\frac{i}{c}+\frac{a_{1} j}{c_{1}}\right) .
$$

Since the numbers $a_{1}$ and $c_{1}$ are coprime, the transformations

$$
\sigma(c)=\frac{1}{c} \sum_{i \bmod d} \sum_{j \bmod c_{1}} e\left(\frac{i}{c}+\frac{j}{c_{1}}\right)=\frac{1}{c} \sum_{k \bmod c} e\left(\frac{k}{c}\right)
$$

lead to the formula

$$
\sigma(c)=\left\{\begin{array}{lll}
1 & \text { if } \quad c=0 \\
0 & \text { if } \quad c \neq 0
\end{array}\right.
$$

Formulas (4.33) and (4.34) imply that

$$
f_{\overrightarrow{\mathbb{Z}}}(\lambda)=0 \quad \text { if } \quad c>1 .
$$

Consequently, it only remains to consider the case where $c=1$. Since, by (4.2), the function $f_{\overrightarrow{\mathbb{Z}}}(\lambda)$ has period 1 ,

$$
f_{\overrightarrow{\mathbb{Z}}}(\lambda+1)=f_{\overrightarrow{\mathbb{Z}}}(\lambda)
$$

without loss of generality we may assume that $a=0$, and then we have $\lambda=b \tau$ by (4.16). Using this relation and formula (4.33), we deduce the formula

$$
f_{\overrightarrow{\mathbb{Z}}}(\lambda)=e\left(b \tau^{2}\right) I_{2}(b \tau, 0,1),
$$

where

$$
I_{2}(b \tau, 0,1)=\int_{0}^{1} e(-\{\Delta+\tau\} b \tau) d \Delta=\int_{0}^{1} e(-\Delta b \tau) d \Delta=\frac{\sin (\pi b \tau)}{\pi b \tau} \exp (-\pi i b \tau) .
$$

Thus, for the amplitude we have the formula

$$
f_{\overrightarrow{\mathbb{Z}}}(\lambda)=\frac{\sin (\pi b \tau)}{\pi b \tau} \exp (-3 \pi i b \tau)
$$

if $c=1$.

Theorem 4.1. The set $\overrightarrow{\mathbb{Z}}=\left\{\vec{N}=F_{1} \circ N ; N \in \mathbb{Z}\right\}$ of Fibonacci-even numbers has the spectrum

$$
\Lambda_{\overrightarrow{\mathbb{Z}}}=\mathbb{Z}[\tau]=\{a+b \tau ; a, b \in \mathbb{Z}\} .
$$

For $\lambda$ in the spectrum $\Lambda_{\overrightarrow{\mathbb{Z}}}$, the amplitude $f_{\overrightarrow{\mathbb{Z}}}(\lambda)$ (see (4.2) $)$ is calculated by formula (4.37). 
Proof. Relation (4.38) for the spectrum $\Lambda_{\vec{Z}}$ follows from (4.10) and (4.35), and formula (4.37) was proved above.

Remark 4.1. In Theorem 4.1, we use the rigorous definition of the spectrum in the Fourier expansion (4.1); i.e., it is a maximal subset $\Lambda_{\overrightarrow{\mathbb{Z}}}$ in $\mathbb{R}$ such that $f_{\overrightarrow{\mathbb{Z}}}(\lambda) \neq 0$ for all $\lambda \in \Lambda_{\overrightarrow{\mathbb{Z}}}$.

Remark 4.2. The method of torus windings used above for finding the spectrum $\Lambda$ is applicable to more general quasilattices of the form

$$
\mathcal{F}_{\omega}=\left\{a_{N, \omega}=\omega_{1} N+\omega_{2}[(N+1) \tau] ; N \in \mathbb{Z}\right\},
$$

where $\omega=\left(\omega_{1}, \omega_{2}\right)$ is a vector in $\mathbb{R}^{2}$. For example, in these terms the Fibonacci quasilattice (0.2) and the set of Fibonacci-even numbers (0.12) considered above have the form $\mathcal{F}=\mathcal{F}_{(1, \tau)}$ and $\overrightarrow{\mathbb{Z}}=\mathcal{F}_{(1,1)}$, respectively.

\section{REFERENCES}

[1] B. K. Vaŭnshtĕn, Modern crystallography. Vol. 2, "Nauka", Moscow, 1980. (Russian)

[2] V. G. Zhuravlev, Rauzy tilings and bounded remainder sets on a torus, Zap. Nauchn. Sem. S.Peterburg. Otdel. Mat. Inst. Steklov. (POMI) 322 (2005), 83-106; English transl., J. Math. Sci. (New York) 137 (2006), no. 2, 4658-4672. MR2138453(2006b:11094)

[3] _ Sums of squares over the Fibonacci o-ring, Zap. Nauchn. Sem. S.-Peterburg. Otdel. Mat. Inst. Steklov. (POMI) 337 (2006), 165-190; English transl., J. Math. Sci. (New York) 143 (2007), no. 3, 3108-3123. MR 2271962 (2007i:11030)

[4] _ One-dimensional Fibonacci tilings, Izv. Ross. Akad. Nauk Ser. Mat. 71 (2007), no. 2, 89-122; English transl., Izv. Math. 71 (2007), no. 2, 307-340. MR2316983 (2008i:11042)

[5] - One-dimensional Fibonacci quasilattices and their application to the Euclidean algorithm and Diophantine equations, Algebra i Analiz 19 (2007), no. 3, 151-182; English transl., St. Petersburg Math. J. 19 (2008), no. 3, 431-454. MR2340709 (2008f:11003)

[6] V. G. Zhuravlev and A. V. Maleev, Diffraction of two-dimensional quasiperiodic Rauzy tiling, crystallography (to appear). (Russian)

[7] L. Kuipers and H. Niederreiter, Uniform distribution of sequences, John Wiley and Sons, New York, 1974. MR0419394(54:7415)

[8] Yu. V. Matiyasevich, The connection between Hilbert's tenth problem and systems of equations between words and lengths, Zap. Nauchn. Sem. Leningrad. Otdel. Mat. Inst. Steklov. (LOMI) 8 (1968), 132-144; English transl., Sem. in Math. V. A. Steklov Math. Inst., Leningrad 8 (1970), 61-67. MR0246772 (40:41)

[9] _ Two reductions of Hilbert's tenth problem, Zap. Nauchn. Sem. Leningrad. Otdel. Mat. Inst. Steklov. (LOMI) 8 (1968), 145-158; English transl., Sem. in Math. V. A. Steklov Math. Inst., Leningrad 8 (1970), 68-74. MR0246773 (40:42)

[10] B. Adamczewski, Répartitions des suites $(n \alpha)_{n \in \mathbb{N}}$ et substitutions, Acta Arith. 112 (2004), 1-22. MR 2040589 (2005f:11158)

[11] T. C. Brown and P. J.-S. Shiue, Sums of fractional parts of integer multiples of an irrational, J. Number Theory 50 (1995), 181-192. MR1316813 (96c:11087)

[12] N. P. Fogg, Substitutions in dynamics, arithmetics and combinatorics, Lecture Notes in Math., vol. 1794, Springer-Verlag, Berlin, 2002. MR1970385 (2004c:37005)

[13] A. Hof, On diffraction by aperiodic structures, Comm. Math. Phys. 169 (1995), 25-43. MR1328260 (97i:82079)

[14] C. Janot, Quasicrystals, Clarendon Press, Oxford, 1994.

[15] D. E. Knuth, Fibonacci multiplication, Appl. Math. Lett. 1 (1988), 57-60. MR0947168 (89f:11031)

[16] J. Lagarias, Mathematical quasicrystals and the problem of diffraction, Directions in Mathematical Quasicrystals, CRM Monogr. Ser., vol. 13, Amer. Math. Soc., Providence, RI, 2000, pp. 61-93. MR $1798989(2001 \mathrm{~m}: 52032)$

[17] Y. Meyer, Algebraic numbers and harmonic analysis, North-Holland Math. Library, vol. 2, NorthHolland Publ. Co., Amsterdam-London, 1972. MR0485769 (58:5579)

[18] R. V. Moody, Meyer sets and their duals, The Mathematics of Long-Range Aperiodic Order (Waterloo, ON, 1995) (R. V. Moody, ed.), NATO Adv. Sci. Inst. Ser. C Math. Phys. Sci., vol. 489, Kluwer Acad. Publ., Dordrecht, 1997, pp. 403-441. MR1460032(98e:52029)

[19] _ Model sets: A survey, From Quasicrystals to More Complex Systems (F. Alex, F. Dénoyer, and J. P. Gazeau, eds.), EPD Science, Les Ulis; Springer-Verlag, Berlin, 2000, pp. 145-166. 
[20] M. Schlottmann, Cut-and-project sets in locally compact abelian groups, Quasicrystals and Discrete Geometry (Toronto, ON, 1995) (J. Patera, ed.), Fields Inst. Monogr., vol. 10, Amer. Math. Soc., Providence, RI, 1998, pp. 247-264. MR1636782 (99f:52021)

[21] _ Generalized model sets and dynamical systems, Directions in Mathematical Quasicrystals (M. Baake and R. V. Moody, eds.), CRM Monogr. Ser., vol. 13, Amer. Math. Soc., Providence, RI, 2000, pp. 143-159. MR.1798991 (2001k:52035)

[22] A. V. Shutov, New estimates in the Hecke-Kesten problem, Analytic and Probabilistic Methods in Number Theory (E. Manstavičius et al., eds.), TEV, Vilnius, 2007, pp. 190-203. MR2397152

Vladimir State Pedagogical University, Av. Stroiteleǐ 11, Vladimir 600024, Russia

E-mail address: vzhuravlev@mail.ru

Received 5/JUN/2007

Translated by N. B. LEBEDINSKAYA 